\title{
A Case Report of a Patient With Liver, Psoas Muscle and Porta Hepatis Abscesses
}

Eugene Kofi Essandoh, MD

\section{Case Report}

A 26-year-old African American male with a history of HIV and CD4 count of 507 on HAART was sent to the Emergency Department after a biopsy of a psoas muscle collection demonstrated pustular drainage. The patient reported that approximately three weeks prior to presentation he was found to have elevated liver function tests on routine labs at his primary care office. Subsequently, the patient was sent for an abdominal ultrasound and CAT scan which showed a confluence of lymph node surrounding the porta hepatis as well as a psoas muscle collection. In review of systems, he reported chronic left-sided weakness and occasional muscle tightness in both legs. $\mathrm{He}$ denied weakness on the right side, as well as, fevers, chills, cough or shortness of breath. The physical exams was unremarkable except for 4/5 weakness in the left lower extremity. Radiographic imaging (Figure 1) revealed fluid collection in the psoas muscle and abscesses in the liver and spleen.

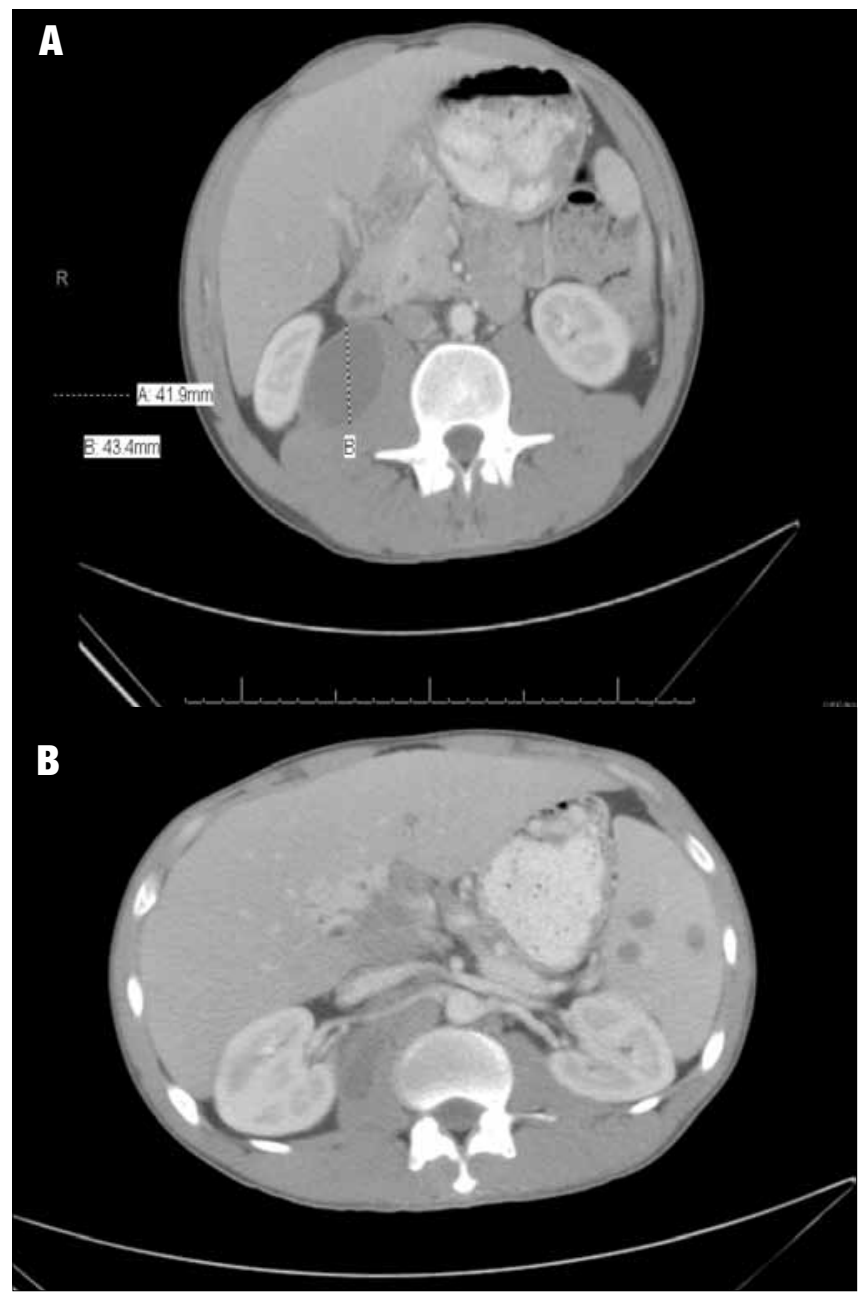

Figure 1. A: Psaos muscle with fluid collection $B:$ Spleen and liver with abscesses
The patient was admitted to the hospital and started on vancomycin. His abscess was drained but the culture did not reveal any organisms. In order to obtain a fluid sample, surgery performed an exploratory laparoscopy of the fluid collection. The direct smear showed acid fast bacilli and the PPD demonstrated induration. Based on these findings, patient was diagnosed with tuberculosis and started on appropriate therapy.

\section{Discussion}

Extrapulmonary tuberculosis (EPTB) refers to tuberculosis infections of organs other than the lung. Affected patients usually have a positive tuberculin skin test and a normal chest $\mathrm{x}$-ray. Tuberculolsis can affect almost all organs. Common sites of infection include the lymph nodes, liver, spleen, psoas muscle, adrenal gland, kidney and osteoarticular areas. A resurgence of tuberculosis in USA occurred in the early 1980's which coincided with the emergence of HIV/AIDS. Though the incidence of the disease is in decline in the US, the global prevalence of the disease is estimated at $32 \%$. The pattern of the disease has changed over the years. Reports demonstrate an increased incidence of disseminated and extrapulmonary TB associated with HIV/ AIDS as well as other immunosuppressed disease-states. ${ }^{1}$

The diagnosis of extrapulmonary TB especially without concomitant pulmonary $\mathrm{TB}$ can be elusive. It requires a high index of suspicion based on the patient's history and risk factors. Clinical clues that should increase suspicion for EPTB are ascites with lymphocyte predominance and negative bacterial cultures; chronic lymphadenopathy; HIV infection; unexplained pericardial effusion or calcification; CSF lymphocytes with elevated protein and low glucose; and exudative pleural effusion with numerous lymphocytes and negative bacteria. A negative acid-fast smear does not exclude the diagnosis. Extra diagnostic tests such as a biopsy or PCR may be necessary in the diagnosis of certain types of EPTB. ${ }^{2}$

Musculoskeletal involvement accounts for approximately 35\% of cases of EPTB. It mostly affects the spine (Pott's disease) and sometimes articular joints. Paraspinal and psoas muscles abscesses can develop through local extension from a spinal abscess or as a primary site. This group of patients may present with symptoms of lower extremity weakness and localizing back pain. A bone, synovial or muscle biopsy for AFB smear and culture is necessary to make definite diagnosis. Patients with hepatic tuberculosis presents with abnormal liver function test. Clinically, they may have symptoms such as right upper quadrant tenderness, nausea and vomiting. The physical exam may reveal hepatomegaly.

The adrenal glands are rarely affected and even when they are, it rarely results in severe adrenal insufficiency. Less than $3 \%$ of TB of the adrenal glands resulted in decreased function of the 
adrenal gland. ${ }^{3}$ Tuberculosis rarely affects the myocardium but when it affects the heart, it usually results in pericarditis. Seeding of $\mathrm{TB}$ in the brain can produce meningitis and tuberculomas. In a study involving TB patients, about 1 out of 5 was found to have TB meningitis or tuberculomas. ${ }^{2}$

In terms of the lymphatic system, TB most commonly affects the cervical nodes. However, axillary, inguinal, mediastinal and mesenteric nodes can be affected as well. Lymph nodes in symptomatic patients are firm, nontender and discrete but gradually evolve into fluctuant nodes. Diagnosis is made by excision biopsy with AFB staining or culture. FNA has a low yield in immunocompetent patients. One can also perform PCR on a lymph node aspirate.

\section{Reference}

1. Marjorie P. Golden, MD, Holenarasipur Vikram, M.D. Extrapulmonary tuberculosis: An Overview. American Family Physician

2. Maartens G; Wilcox PA; Benatar SR. Miliary Tuberculosis: rapid diagnosis, hematologic abnormalities, and outcome in 109 treated adults. AmJ Med 1990 Sep; 89 (3): 291-6.

3. Barnes DJ, Naraqi S; Temu P; Turtle JR. Adrenal function in patients with active tuberculosis. Thorax 1989; 44:422-424.

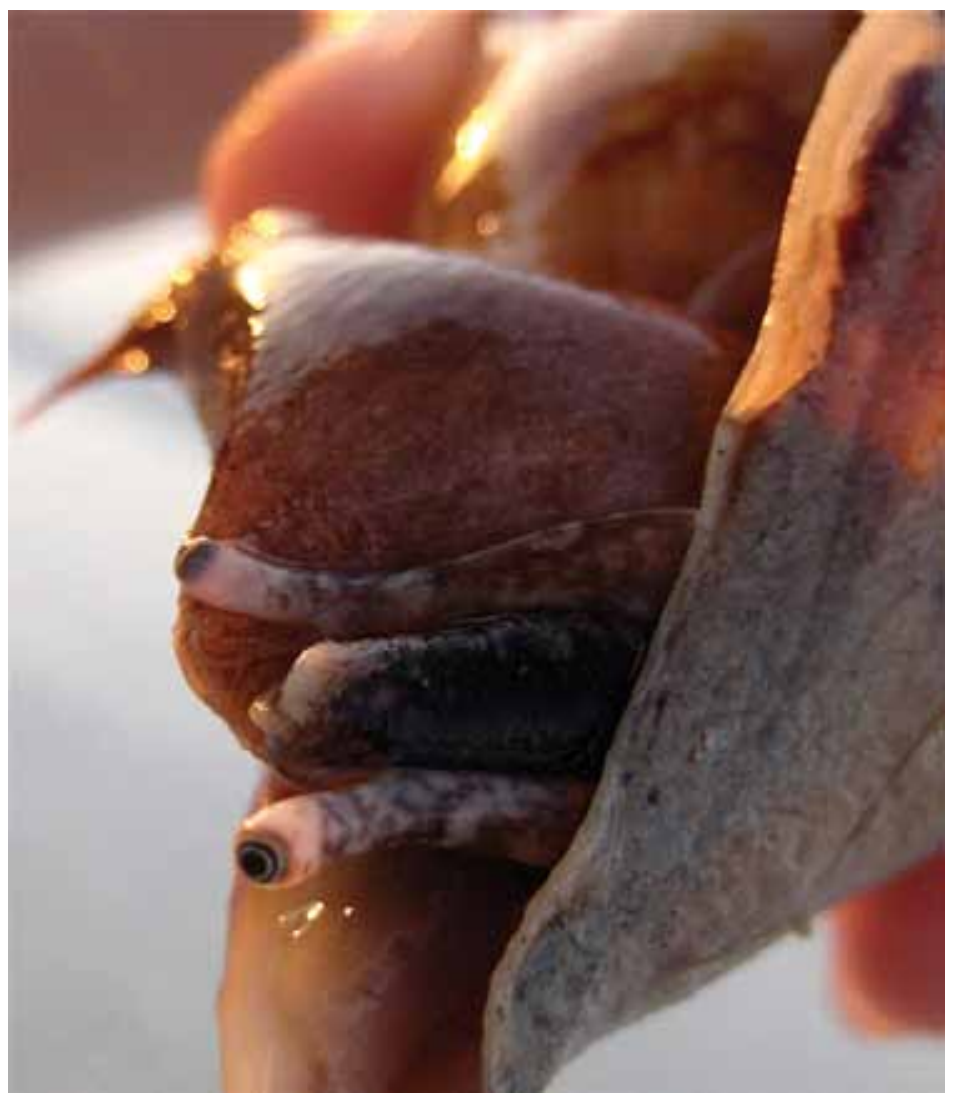

"Eyes"

Photograph by Cecilia Kelly, MD 